\title{
Diverse roles of miR-29 in cancer (Review)
}

\author{
HESONG JIANG $^{1,2^{*}}$, GUANG ZHANG ${ }^{1,2,4^{*}}$, JUN-HUA WU ${ }^{1}$ and CHUN-PING JIANG ${ }^{2,3}$ \\ ${ }^{1}$ Jiangsu Key Laboratory of Molecular Medicine, Medical School, Nanjing University, Nanjing, Jiangsu 210093; \\ ${ }^{2}$ Department of Hepatobiliary Surgery, The Affiliated Drum Tower Hospital, Medical School, Nanjing University, \\ Nanjing, Jiangsu 210008; ${ }^{3}$ The Drum Tower Clinical Medical College, Nanjing University of Chinese Medicine, \\ Nanjing, Jiangsu 210046; ${ }^{4}$ Department of Hepatobiliary Surgery, Drum Tower Medical College \\ of Nanjing Medical University, Nanjing, Jiangsu 210008, P.R. China
}

Received December 9, 2013; Accepted January 17, 2014

DOI: $10.3892 /$ or.2014.3036

\begin{abstract}
RNAs (miRNAs) are non-coding RNAs which have the capacity to regulate gene expression at the post-transcriptional level, and have emerging as key factors involved in cancer at all stages ranging from initiation to metastasis. In the present review, we summmarize the diverse roles of the microRNA-29 (miR-29) family in cancer. First, we present a concise introduction to the miR-29 family and the expression profile of miR-29 in various cancer types. We next highlight the upstream regulatory pathway of miR-29 and describe the relationship between miR-29 and cancer in detail. As a tumor suppressor, miR-29 restrains cancer progression by promoting tumor cell apoptosis, by suppressing DNA methylation of tumor-suppressor genes, by reducing proliferation of tumors and by increasing chemosensitivity. However, as a tumor promoter, miR-29 mediates epithelial-mesenchymal transition (EMT) and promotes metastasis in breast cancer
\end{abstract}

Correspondence to: Dr Jun-Hua Wu, Jiangsu Key Laboratory of Molecular Medicine, Medical School, Nanjing University, Nanjing, Jiangsu 210093, P.R. China

E-mail: njjhs2007@163.com

Professor Chun-Ping Jiang, The Affiliated Drum Tower Hospital, Medical School, Nanjing University, Nanjing, Jiangsu 210008, P.R. China

E-mail: chunpunjiang@163.com

${ }^{*}$ Contributed equally

Abbreviations: EZH2, enhancer of zeste homolog 2; YY1, Yin Yang 1; HDACs, histone deacetylases; CEBPA, CCAAT enhancer binding protein $\alpha$; CDK6, cyclin-dependent kinase 6; FLT3, Fms-like tyrosine kinase-3; GATA3, GATA binding protein 3; WWOX, WW domain-containing oxidoreductase; VEGFA, vascular endothelial growth factor $\alpha$; ANGPTL4, angiopoietin-like 4; PDGF, platelet-derived growth factor; LOX, lipoxygenase; MMP9, matrix metallopeptidase 9; ITGA6, integrin $\alpha 6$; ITGB1, integrin $\beta 1$; TGF, transforming growth factor

Key words: miR-29, cancer, apoptosis, epithelial-mesenchymal transition, diagnosis, prognosis, biomarker and colon cancer. Finally, we suggest that miR-29 represents a novel diagnostic and prognostic biomarker or a therapeutic target for cancer. Our review highlights the diverse relationship between miR-29 and cancer (particularly digestive system neoplasms). Further research of miR-29 in cancer is warranted.

\section{Contents}

1. Introduction

2. A brief introduction to the microRNA-29 family

3. Expression of miRNA-29 in cancers

4. Upstream regulation of miR-29 expression in cancer

5. Function of miR-29 in cancer

6. Clinical implications

7. Conclusions and prospects

\section{Introduction}

Cancer represents one of the major public health issues and is a leading cause of worldwide mortality. Throughout the past 10 years, the cancer-related death rate was $25 \%$ of the total mortality; therefore, overcoming cancer is an urgent priority (1). After unremitting research efforts, it has been definitely proven that the initiation of cancer is the result of the deregulation of oncogenes and tumor-suppressor genes. Recently, microRNAs (miRNAs) have been identified as novel regulators of oncogenes and tumor-suppressor genes. Researchers believe that miRNAs will become a new modality for both the diagnosis and treatment of cancers $(2,3)$.

MiRNAs are a large class of small, non-coding, singlestranded RNAs, 19-23 nucleotides in length, and constitute a novel class of gene regulators (4). These single-stranded miRNAs bind to complementary sites on the 3' untranslated region (UTR) of target genes, and consequently regulate posttranscriptional gene expression by mRNA degradation and translational repression (5). By targeting multiple transcripts, miRNAs are involved in biological processes including cell differentiation, proliferation, apoptosis and metastasis (6).

There is increasing evidence that miRNAs widely participate in the regulation of oncogenes (7) or tumor suppressors (8) 


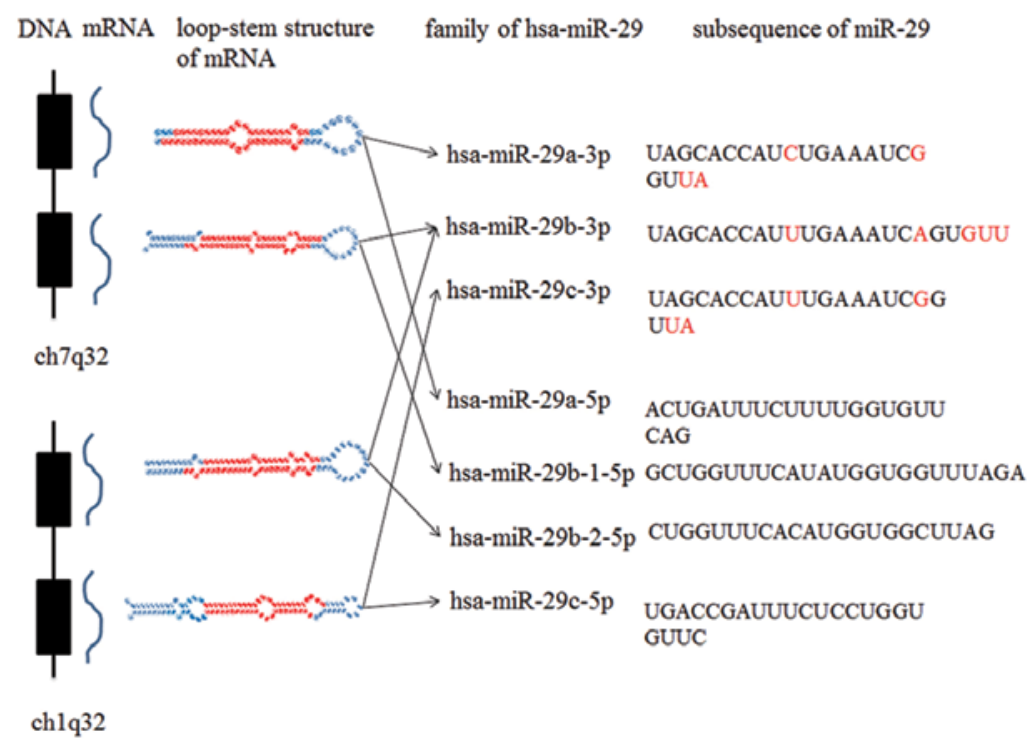

Figure 1. The loop-stem structure of mRNA and the sequence of the miR-29 family. The loop-stem structure of mRNAs, miR-29-3p or -5p, are indicated in red. In overwhelmingly predominant miR-29-3p, nucleotides that differ among the miR-29 family members are indicated in red.

in cancer. Previous research has shown that miRNA-21 functions as an oncogene by inhibiting tumor suppressors such as phosphatase and tensin homolog (PTEN) (9) in Kazakh's esophageal squamous cell carcinoma and programmed cell death-4 (PDCD4) in human hepatocellular carcinoma (10). Whereas, miR-15 and miR-16 induce apoptosis and act as tumor suppressors by targeting $\mathrm{Bcl}$ (11). It was recently suggested that the miR-29 family is involved in many diseases and pathophysiological processes. For example, miR-29 was found to play an important role in aneurysm formation (12), systemic sclerosis (13), preeclampsia (14) and irritable bowel syndrome (15), and also was found to have an impact on pathophysiological processes of liver fibrosis (16), cardiac fibrosis (17), renal fibrosis (18) and myocardial ischemiareperfusion injury (19). More and more research indicates that miR-29 participates in the processes of apoptosis, proliferation and epithelial-mesenchymal transition (EMT) in cancer. Here, we review the relationship between miR-29 and cancer in detail.

\section{A brief introduction to the microRNA-29 family}

MicroRNA is produced by shearing the arm of the stem-loop or hairpin which is a general shape of corresponding microRNA precursor or pre-miRNA. Although a mature miRNA may only come from one arm of pre-miRNA, the $3^{\prime}(3 p)$ or the $5^{\prime}$ (5p) arm is predominantly processed. In terms of miR-29, the $3 p$ arm of the miR-29 precursor is clipped as a preferential overwhelming product (miR-29 or miR-29-3p), although the $5 p$ arm (miR-29* or miR-29-5p) also objectively exists. Thus, the human miRNA-29 family has three main members including hsa-miR-29a, hsa-miR-29b and hsa-miR-29c (Fig. 1).

These three miR-29s are distributed on two chromosomes: miR-29b2/miR-29c located on chromosome 1q32 and miR-29b1/miR-29a located on chromosome 7q32 (20). Among the members of the miR-29 family, miR-29a was firstly discovered by Lagos-Quintana et al (21). Subsequent large-scale cloning studies revealed the sequence of mature miR-29a. Mourelatos et al (22) identified miR-102 located on chromosome 7 primordially, which was known as miR-29b because of the isogeny between human miR-102 and mouse miR-29b. Researchers then found the same sequence on chromosome 1q32. Researchers distinguished them as miR-29b1 located on chromosome 7q32 and miR-29b2 located on chromosome 1q32. With the development of technology, miR-29c was found by small RNA library sequencing (23).

\section{Expression of miRNA-29 in cancers}

Transcriptional profiling studies of miRNA expression across tumor tissues or cancer cell lines have revealed that miR-29 is downregulated in the majority of cancers and upregulated in the minority. As documented in Table I, aberrant expression of miR-29 and the oncogenic or tumor-suppressive functions of miR-29 have been extensively investigated in many types of cancers.

Downregulation of miR-29 has been found in many solid tumors and hematologic neoplasms by transcript profiling or northern blot analysis. In the respiratory system, miR-29 is expressed at a lower level in lung cancer tissue when compared with that in normal lung tissue (24). Fabbri et al (25) demonstrated that expression of miR-29a, miR-29b, and miR-29c is downregulated in NSCLCs. Plaisier et al (26) found that the miR-29 family (miR-29a, miR-29b and miR-29c) inhibited specific genes associated with invasion and metastasis of lung adenocarcinoma. In the nervous system (NS), miR-29 was found to be downregulated in NS neoplasms such as glioblastomas (27) and neuroblastoma (28). In the muscular and skeletal systems, miR-29 was found to be significantly downregulated in osteoblastoma (29) and rhabdomyosarcoma (30). MiR-29a induced osteoblastic cell apoptosis by silencing Bcl-2 and Mcl-1 and inducing E2F1 and E2F3 expression (31). In the genitourinary system, all three isoforms of miR-29 (a, $\mathrm{b}$ and c) are highly expressed in normal tissues. However, 
Table I. Expression of miR-29 in cancer.

\begin{tabular}{|c|c|c|c|}
\hline Organizational systems & Tumor tissue & Regulation of miR-29a & Refs. \\
\hline \multirow[t]{3}{*}{ Respiratory system } & Lung cancer & Downregulation & $(24)$ \\
\hline & Non-small cell lung cancer & Downregulation & $(25])$ \\
\hline & Lung adenocarcinoma & Downregulation & $(26)$ \\
\hline \multirow[t]{5}{*}{ Digestive system } & Esophageal carcinoma & Downregulation & $(51)$ \\
\hline & Stomach cancer & Downregulation & $(50)$ \\
\hline & Hepatocellular carcinoma & Downregulation & $(52,53)$ \\
\hline & Cholangiocarcinoma & Downregulation & $(54)$ \\
\hline & Colon cancer & Upregulation & $(56)$ \\
\hline \multirow[t]{2}{*}{ Nervous system } & Glioblastomas & Downregulation & (27) \\
\hline & Neuroblastoma & Downregulation & $(28)$ \\
\hline \multirow[t]{2}{*}{ Muscular and skeletal systems } & Osteoblastoma & Downregulation & $(29,31)$ \\
\hline & Rhabdomyosarcoma & Downregulation & $(30)$ \\
\hline \multirow[t]{3}{*}{ Genitourinary system } & Bladder cancer & Downregulation & $(32,33)$ \\
\hline & Prostate cancer & Downregulation & $(34)$ \\
\hline & Renal carcinoma & Downregulation & $(38)$ \\
\hline \multirow[t]{3}{*}{ Female reproductive system } & Ovarian cancer & Downregulation & $(35,36)$ \\
\hline & Endometrial cancer & Downregulation & $(37)$ \\
\hline & Breast cancer & Upregulation & $(47)$ \\
\hline \multirow[t]{6}{*}{ Hematopoietic system } & Acute lymphoblastic leukemia & Downregulation & (39) \\
\hline & Acute myeloid leukemia (AML) & Downregulation & $(40)$ \\
\hline & Chronic lymphocytic leukemia & Downregulation & $(41)$ \\
\hline & $\mathrm{NPMc}^{+} \mathrm{AML}$ & Upregulation & $(23)$ \\
\hline & Diffuse large B lymphoma & Upregulation & $(49)$ \\
\hline & Mantle cell lymphoma & Downregulation & $(42)$ \\
\hline \multirow[t]{3}{*}{ Other systems } & Basal cell carcinoma & Downregulation & $(44)$ \\
\hline & Nasopharyngeal carcinomas & Downregulation & $(45,46)$ \\
\hline & Malignant pleural mesothelioma & Upregulation & $(48)$ \\
\hline
\end{tabular}

${ }^{a}$ miR-29 level in cancers compared with that in corresponding normal tissue. $\mathrm{NPMc}^{+} \mathrm{AML}$, acute myeloid leukemia bearing cytoplasmic nucleophosmin.

they are downregulated in genitourinary tumors including bladder $(32,33)$, prostate $(34)$, ovarian $(35,36)$, endometrial cancer (37) and renal carcinoma (38). In the hematopoietic system, miR-29 is downregulated in acute lymphoblastic leukemia (ALL) (39), acute myeloid leukemia (AML) (40), chronic lymphocytic leukemia (CLL) (41) and mantle cell lymphoma (MCL) (42) when compared with normal cases (43). In addition, miR-29 was found to be significantly downregulated in basal cell carcinoma (BCC) when compared with normal skin (44). Expression of miR-29 was also downregulated in nasopharyngeal carcinomas $(45,46)$.

Although miR-29 is downregulated in the vast majority of cancers, it is also upregulated in a minority of cancers. MiR-29 is also expressed at a high level in tumor tissues or serum from breast cancer patients when compared to its expression in normal cells or serum from healthy individuals (47). The upregulated expression of miR-29 was also detected in several rare diseases such as malignant pleural mesothelioma (48) and diffuse large B lymphoma (49). Notably, although miR-29 is downregulated in ALL, Garzon et al (39) found that miR-29 was highly expressed in acute myeloid leukemia bearing cytoplasmic nucleophosmin $\left(\mathrm{NPMc}^{+} \mathrm{AML}\right)$. This phenomenon indicates that there are wide differences in the expression profile of miR-29 in subtypes of the same cancer.

The differential expression of miR-29 has also been noted in the digestive system. The expression of miR-29 is downregulated in gastric carcinoma (50). A low level of miR-29 caused loss of inhibition of cyclin E expression, resulting in esophageal carcinoma cell cycle activation (51). Several studies have demonstrated that high expression of miR-29 induces hepatocellular carcinoma cell apoptosis, while downregulation of miR-29 increases HCC tumorigenicity and metastasis $(52,53)$. Mott et al (54) found a high level of miR-29 in normal cholangiocytes by northern blot analysis. It is notable that downregulation of miR-29 was associated with upregulated Mcl-1 protein in cholangiocarcinoma. In contrast, upregulation of miR-29a was observed in the plasma of colorectal cancer (CRC) patients. This, it may be a potential biomarker to 


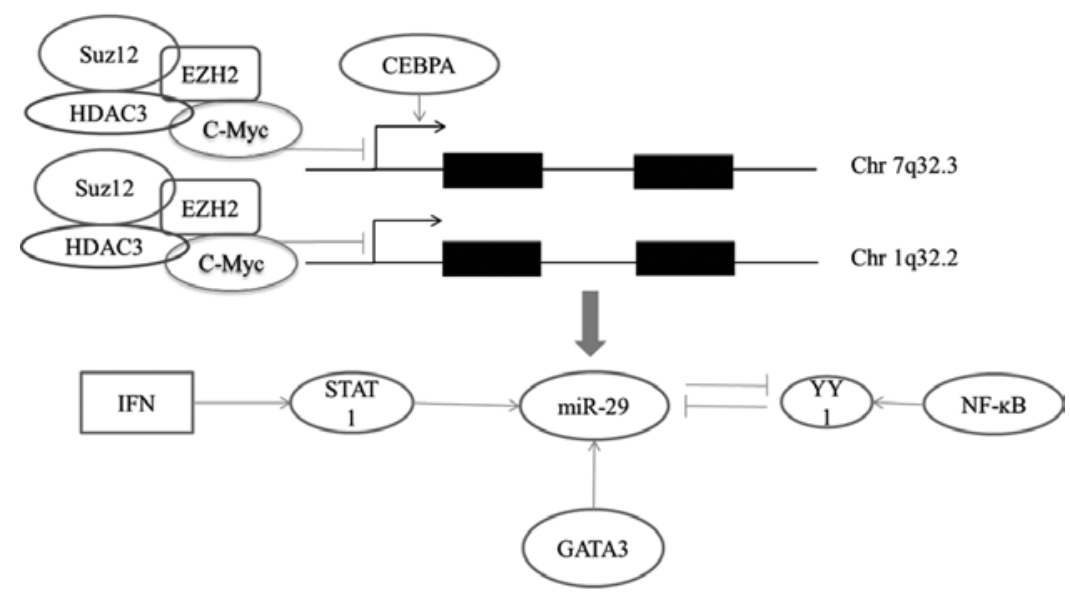

Figure 2. Transcriptional regulation of miR-29.

differentiate CRC from controls (55). Wang and Gu (56) found that serum miR-29a was also significantly higher in patients with colorectal liver metastasis when compared with the serum level in CRC patients. It appears that miR-29 is associated with the degree of malignancy in colorectal cancer.

\section{Upstream regulation of miR-29 expression in cancer}

miR-29 is generated by non-coding mRNA splicing and is regulated by many signaling molecules such as Myc, EZH2, YY1, HDAC and GATA3. These regulatory proteins control expression of miR-29 mainly in the early stages (Fig. 2).

In this regard, in CLL, histone deacetylases (HDACs) can downregulate the level of miR-29 (57). Intensive research has demonstrated that HDAC3 (one member of the HDACs) is a component part of the HDAC3-EZH2 complex, and MYC suppresses miR-29 expression through the HDAC3-EZH complex (58). There are two other miRNAs (miR-26a and miR-494) indirectly involved in the MYC-EZH2-miR-29 axis. MYC upregulates EZH2 by repressing miR-26a which is an inhibitor of EZH2, and EZH2 suppresses miR-494 which could inhibit MYC. Through miR-26a and miR-494, MYC and EZH2 form a positive feedback loop. Next, EZH2 combined with HDAC3 results in a low level of miR-29 expression (58-60).

Wang et al (61) described the regulation and function of miR-29 in myogenesis and rhabdomyosarcoma (RMS) and found that miR-29 participates in the regulatory circuit involving NF- $\kappa \mathrm{B}$ and YY1. In RMS cells and primary tumors, the NF- $\mathrm{B}-\mathrm{YY} 1$ pathway is activated while miR-29 is epigenetically silenced. miR-29 is repressed by YY1 which can be activated by $\mathrm{NF}-\kappa \mathrm{B}$. On the other hand, miR-29 can also target its repressor YY1 in turn. Wang et al (61) also found that miR-29 and YY1 suppress each other; miR-29 blocks YY1 translation and YY1 inhibits miR-29 transcription, and constructs a negative feedback loop. NF- $\kappa \mathrm{B}$ further regulates this loop which directly activates YY1 and indirectly controls miR-29.

In addition to MYC and YY1, there are other regulators of miR-29. The hematopoietic key transcription factor CEBPA can induce miR-29 expression. Notable, since CEBPA is located on chromosome 7q, CEBPA selectively upregulates
miR-29a/b1 expression on chromosome 7q32.3, while it cannot affect miR-29b2/c on chromosome 1q32.2 (62).

As previously confirmed, IFN is an agonist of signal transducer and activator of transcription 1 (STAT1) and CDK6, two targets of miR-29. Schmitt et al (63) found that STAT1 upregulated the tumor suppressing miR-29 in melanoma cells. They also observed that increased expression of interferon- $\gamma$ (IFN- $\gamma$ ) resulted in G1-arrest of melanoma cells involving CDK6 repression.

Recently, researchers have demonstrated that, in breast cancer, GATA3, a transcription factor, suppresses metastasis and alters the tumor microenvironment by inducing miR-29b expression. As a tumor-suppressor gene, GATA3 is lost in breast cancer, and is correlated with a worse patient prognosis $(64,65)$.

\section{Function of miR-29 in cancer}

As a newly discovered tumor suppressor in most cancers, miR-29 can downregulate oncogenes and/or upregulate tumor suppressors; thus, miR-29 can induce apoptosis in cancer cells and inhibit proliferation and invasiveness of human cancer cells (66). Notably, in some tumors such as breast cancer and colon cancer, miR-29 can induce epithelial-to-mesenchymal transition. In the present review, we present a mechanism by which miR-29 functions (Figs. 3 and 4).

Inhibition of the cancerigenic process. DNA methylation can induce the silencing of genes. Demethylation of oncogenes and methylation of anti-oncogenes are commonly acknowledged cancerigenic processes. DNA methyltransferase-3A (DNMT-3A) and DNA methyltransferase-3B (DNMT-3B), two key enzymes involved in DNA methylation, are frequently upregulated in many types of cancers and are associated with poor prognosis. The miR-29 family (29a, 29b and 29c) has a finely complementary structure to the 3'-UTRs of DNMT-3A and DNMT-3B (60). Fabbri et al (25) found that there are few tumor-suppressor genes, such as PTEN and $W W O X$, expressed in lung cancer cell lines due to methylation of these tumor suppressors. However, when expression of miR-29s was enforced in these lung cancer cell lines, DNA methylation of tumor-suppressor genes was suppressed and tumor-suppressor 


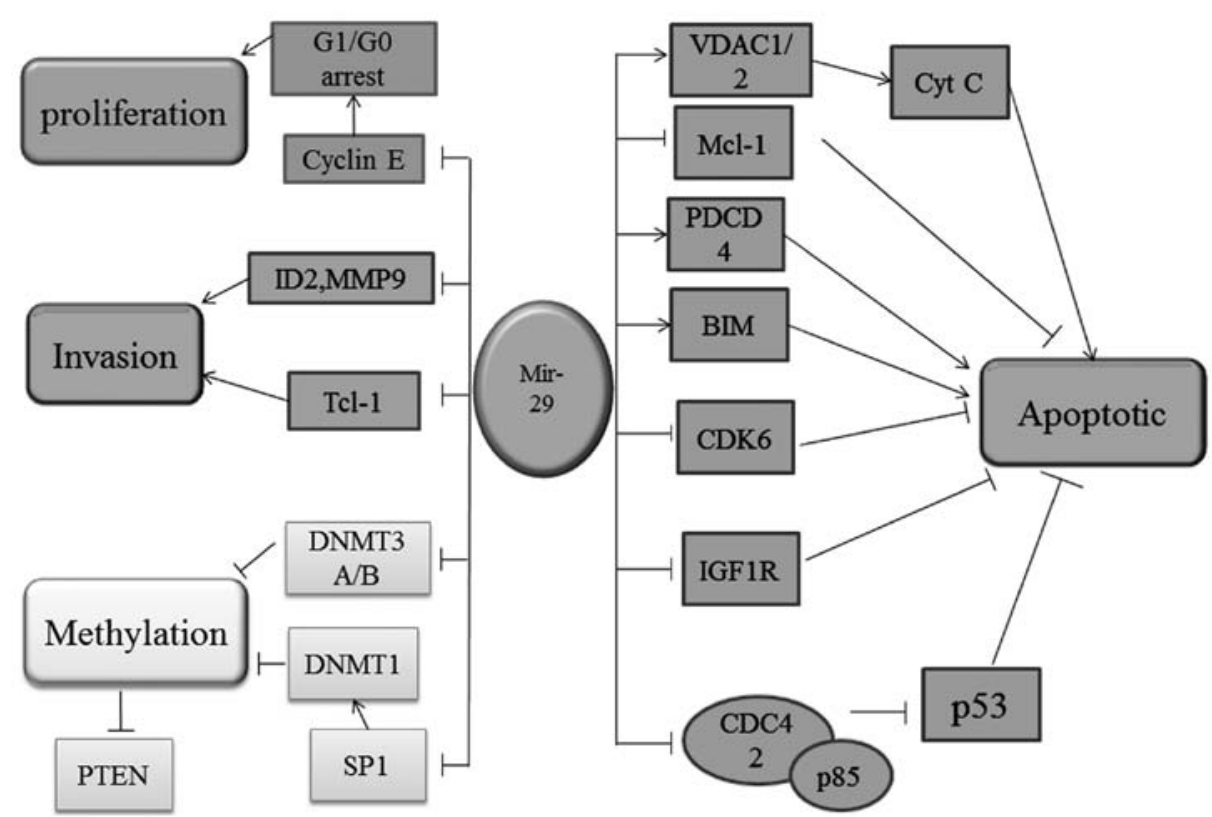

Figure 3. The downstream pathways of miR-29 and the relationship between miR-29 and cancer.

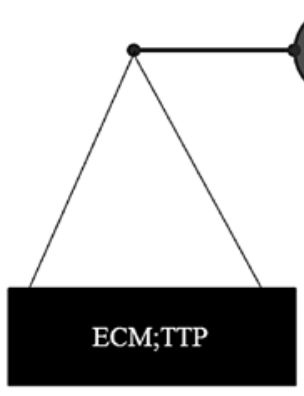

mesenchymal
$-29$

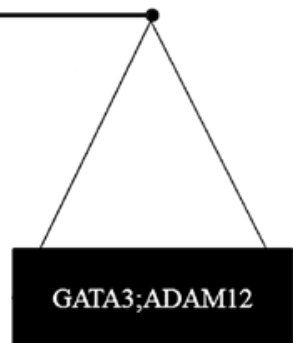

epithelial

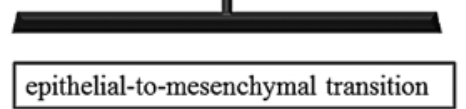

Figure 4. The function of miR-29 in the balance of epithelial-to-mesenchymal transition.

genes were re-expressed. Garzon et al (67) found that miR-29b not only directly combined with DNMT3A and DNMT3B but also indirectly suppressed DNMT1 by Sp1 (a transactivator of the DNMT1 gene). Therefore, a high level of miR-29 induces global DNA hypomethylation, and consequently tumor-suppressor genes are re-expressed. Li et al (68) and Wang et al (69) demonstrated that miR-29b blocked the expression of DNA methyltransferases (Dnmts), and then caused hypomethylation in the PTEN promoter and finally upregulated PTEN gene expression and inhibited the cancerigenic process.

Promotion of cancer cell apoptosis. Promotion of cancer cell apoptosis is one of the most effective strategies for treatment of cancer. Mcl-1 possesses BH1-3, a Bcl2 homology subunit, and has a potent multidomain anti-apoptotic protein of the Bcl-2 (B-cell lymphoma 2) family (53). Endogenous miR-29 directly binds the 3'UTR of Mcl-1 and prevents Mcl-1 protein expression, and promotes tumor cell apoptosis. Unfortunately, miR-29b is downregulated with a synchronous high level of Mcl-2 in cholangiocarcinoma malignant cells. Factitiously enhanced exogenous miR-29b expression endogenously reduces Mcl-1 cellular protein levels and increases the sensitivity of cancer cells to tumor necrosis factor-related apoptosis-inducing ligand (TRAIL) cytotoxicity (54). During the course of apoptosis, mitochondria release apoptogenic factors such as cytochrome $c$ by VDAC, and increasing permeability of VDAC promotes the apoptosis of tumor cells. Recent studies have found that VDAC1 and VDAC2 are targets of miR-29a. Overexpression of miR-29a can lead to a high level of VDAC1 and VDAC2 and increases permeability of VDAC (70). Furthermore, as the classical pathway of apoptosis, miR-29 family members (miR-29a, miR-29b and miR-29c) increase p53 levels and induce apoptosis by a p53-dependent manner. Park et al (71) found that miR-29 family members were able to directly suppress p85a (the regulatory subunit of PI3 kinase) and CDC42 (a Rho family GTPase). p85a and CDC42 regulate 553 negatively, and then induce apoptosis by a p53-dependent signaling pathway. Both miR-29a and miR-29b not only directly target anti-apoptotic genes but also upregulate pro-apoptotic genes, such as BIM (BCL2L11) and the tumor suppressor PDCD4 (72).

Suppression of tumor invasion. Highly invasive tumors are associated with poor prognosis. T-cell leukemia-1 (Tcl1) (one of the cytokines in T cell leukemia) appears to be associated with tumor invasion. Pekarsky et al (73) suggested that Tcll expression in CLL is regulated by a number of miRNAs and also emphasized that miR-29 is the most important one which can reduce the invasiveness of Tcl1-overexpressed CLLs. Another study demonstrated that miR-29b binds to the 3'-UTR 
of inhibitor of DNA binding 1 (ID1) and inhibition of endogenous miR-29b causes an increase in ID1 and MMP9, leading to enhanced tumor cell invasion. In contrast, downregulation of ID1 and MMP9 by stable overexpression of miR-29b could significantly suppress tumor cell invasion. These results suggest that by direct interaction with ID1 and regulation of MMP9, which is a known target of ID1, miR-29b plays an important role in the regulation of tumor cell migration and invasion (74).

However, recent research indicates that in breast cancer miR-29 has an opposing function in regards to invasion. C1QTNF6, SPARC and COL4A2 are targeted by miR-29b and downregulation of their mRNAs was found to contribute to the promotion of the invasive ability of human breast cancer cell line MCF-7 (75). Cochrane et al (76) also found that progestin treatment decreased miR-29, thereby relieving repression of one of its direct targets, the gene encoding ATPase $\mathrm{Na}(+) / \mathrm{K}(+)$ transporting $\beta 1$ polypeptide (ATP1B1), and ATP1B1 inhibits the migration and invasion of breast cancer cells.

Induction of cell cycle arrest to inhibit tumor proliferation. Cyclin $\mathrm{E}$ is a protein which can regulate the cell cycle. Ding et al (51) found that miR-29c was frequently downregulated in ESCC tissues and cells, and artificial upregulation of miR-29 suppressed tumor growth by inducing cell cycle G1/ G0 arrest mainly through downregulation of the expression of cyclin E. Recently, Lee et al (77) found that the 2'-5'-oligoadenylate (2-5A) system, an interferon-regulated RNA decay pathway, activated tumor cell proliferation in vitro as well as tumor growth in a xenograft model, and the miR-29 family suppressed the expression of RNase-L, a key component of the 2'-5'-oligoadenylate (2-5A) system, and finally loss of RNase-L obviously inhibited tumor cell proliferation.

Role in EMT. Based on the above research findings, enhanced miR-29a expression increases the invasive ability of breast cancer cells. Invasion is a result of epithelial-mesenchymal transition (EMT). Gebeshuber et al (78) revealed that miR-29a was upregulated in the process of EMT by comparing metastatic RasXT cells with epithelial EpRas cells, and it could suppress the expression of tristetraprolin (TTP) which is involved in the inhibition of EMT. Furthermore, overexpression of miR-29a inhibited TTP and led to tumor metastasis in cooperation with the oncogenic Ras signaling pathway. Several studies demonstrated that miR-29 could upregulate multiple genes encoding extracellular matrix (ECM) proteins, including collagens, fibrillins and elastin. ECM proteins are motivators in the process of EMT, and miR-29 can induce EMT through these ECM proteins. There is also evidence that miR-29 may regulate MMP2 or Mcl-1, which partly participate in the process of metastasis, yet the underlying mechanisms remain controversial (79-81). In summary, in some situations enhanced miR-29a levels can induce EMT and increase the metastasis of tumors.

In contrast, $\mathrm{Li}$ et al (82) reported that in breast cancer ADAM12 (a breast cancer associated gene) is negatively regulated by miR-29. ADAM12 is strongly associated with invasion and the process of EMT. Overexpression of miR-29 could block EMT by the downregulation of ADAM12. Two recent studies found that GATA3 enhanced the level of miR-29b in breast cancers and conversely loss of miR-29b increased metastasis and promoted EMT. Furthermore, miR-29b inhibited metastasis by targeting a network of pro-metastatic motivators involved in angiogenesis, collagen remodelling and proteolysis, including VEGFA, ANGPTL4, PDGF, LOX and MMP9, and targeting ITGA6, ITGB1 and TGFB, thereby indirectly affecting differentiation and EMT $(64,65)$.

Clinical implications. MiR-29 may be used as a biomarker for cancer diagnosis and may facilitate early diagnosis. Wang and $\mathrm{Gu}$ (56) demonstrated that serum miR-29a was a promising novel marker for early detection of colorectal liver metastasis. Fang et al (49) showed that serum microRNAs were promising biomarkers for diffuse large B cell lymphoma. Zhi et al (83) found that miR-29 can also act as a biomarker of meningioma for early diagnosis. A low level of miR-29c was found to be associated with advanced clinical stages of meningioma and was found to be significantly correlated with higher rates of recurrence in meningioma patients. It has been confirmed that miR-29 and miR-142 are consistently decreased in AML and may function jointly in granulopoiesis and monopoiesis (81). Thus, combined assessment of miR-29 and miR-142 is a more effective diagnostic method for AML. The sensitivity and specificity of this method are 90 and $100 \%$, respectively.

MiR-29s play important roles in tumor stage. WeissmannBrenner et al (84) showed that high expression of miR-29a was associated with a prolonged disease-free survival. In stage I colon cancer, 903 microRNAs tested exhibited differential expression between patients with good prognosis and those with poor prognosis. Notably, miR-29a was found to be upregulated in patients with a favorable prognosis when compared with its expression in patients with poor prognosis in stage II colon cancer.

MiR-29 can also increase the sensitivity of cancer or cancer cells to chemotherapeutic drugs. Blum et al (85) examined the relationship between the clinical response to decitabine and bortezomib treatment and the level of miR-29b. They further revealed that bortezomib can upregulate miR-29b and increase the clinical sensitivity of tumors to decitabine. Mechanistic studies showed that bortezomib upregulates miR-29b and then epigenetically downregulates FLT3. Then downregulated FLT3 leads to conspicuous downregulation of SP1 and destroys the SP1/NF- $\mathrm{BB}$ complex. This study also demonstrated that combination of decitabine and bortezomib in AML is feasibility and effective (86).

MiR-29 can be a prognostic indicator for patients with cancer. High expression of ZAP-70 protein and low expression of the miR-29 family were strongly associated with disease progression in CLL cases harboring the $17 \mathrm{p}$ deletion (87). Zhu et al (88) indicated that a low level of miR-29a was associated with advanced clinical features and poor prognosis of pediatric AML patients, suggesting that miR-29a downregulation may be used as a malignant prognostic marker in pediatric AML. Moreover, the level of miR-29 could be used for estimating the relapse-free and overall survival of pediatric AML patients. They also demonstrated that expression levels of miR-29a in pediatric AML patients were significantly lower in subtype M7 than that in other subtypes. Thus, miR-29 has the potential to be used to classify different subtypes of the same cancer. 


\section{Conclusions and prospects}

With the rapid development of biotechnology, the mysteries of microRNAs are gradually being solved. MicroRNAs have an extremely close relationship with tumors and play an important role in the process of tumor initiation and development. In most cancers, miR-29s act as tumor suppressors by influencing apoptosis, methylation, invasion, proliferation and chemotherapeutic sensitivity. However, it nust be noted that miR-29 acts as a promoter of metastasis. This phenomenon is noted in different types of cancers. We highlight that miR-29 also plays diverse roles in the digestive system. The mechanism of this phenomenon has not been fully explained. Therefore, the relationship between miR-29 and cancer (particularly digestive system neoplasms) require further research.

\section{Acknowledgements}

The present review was supported by the National Natural Science Foundation of China (no. NSFC30801417); the Natural Science Foundation of Jiangsu Province (no. BK2009010); the Doctoral Fund of the Ministry of Education of China (no. RFDP200802841004); the Science Fund of the Ministry of Health of China (no. LW201008); and the Key Project supported by the Medical Science and Technology Development Foundation, Nanjing Department of Health (no. ZKX12011).

\section{References}

1. Siegel R, Naishadham D and Jemal A: Cancer Statistics, 2012 CA Cancer J Clin 62: 10-29, 2012.

2. Cantley LC, Auger KR, Carpenter C, et al: Oncogenes and signal transduction. Cell 64: 281-302, 1991.

3. Levine AJ, Momand J and Finlay CA: The p53 tumour suppressor gene. Nature 351: 453-456, 1991.

4. Chen K and Rajewsky N: The evolution of gene regulation by transcription factors and microRNAs. Nat Rev Genet 8: 93-103, 2007.

5. Bartel DP: MicroRNAs: target recognition and regulatory functions. Cell 136: 215-233, 2009.

6. Lu J, Getz G, Miska EA, et al: MicroRNA expression profiles classify human cancers. Nature 435: 834-838, 2005.

7. He L, Thomson JM, Hemann MT, et al: A microRNA polycistron as a potential human oncogene. Nature 435: 828-833, 2005.

8. He L, He XY, Lim LP, et al: A microRNA component of the p53 tumour suppressor network. Nature 447: U1130-U1116, 2007.

9. Ugalde AP, Ramsay AJ, de la Rosa J, et al: Aging and chronic DNA damage response activate a regulatory pathway involving miR-29 and p53. EMBO J 30: 2219-2232, 2011.

10. Zhu Q, Wang ZM, Hu Y, et al: miR-21 promotes migration and invasion by the miR-21-PDCD4-AP-1 feedback loop in human hepatocellular carcinoma. Oncol Rep 27: 1660-1668, 2012.

11. Cimmino A, Calin GA, Fabbri M, et al: miR-15 and miR-16 induce apoptosis by targeting BCL2. Proc Natl Acad Sci USA 102: 13944-13949, 2005.

12. Boon RA, Seeger T, Heydt S, et al: MicroRNA-29 in aortic dilation: implications for aneurysm formation. Circ Res 109: $1115-1119,2011$.

13. Maurer B, Stanczyk J, Jungel A, et al: MicroRNA-29, a key regulator of collagen expression in systemic sclerosis. Arthritis Rheum 62: 1733-1743, 2010.

14. Li PF, Guo W, Du LL, et al: microRNA-29b contributes to pre-eclampsia through its effects on apoptosis, invasion and angiogenesis of trophoblast cells. Clin Sci 124: 27-40, 2013.

15. Zhou QQ, Souba WW, Croce CM and Verne GN: MicroRNA-29a regulates intestinal membrane permeability in patients with irritable bowel syndrome. Gut 59: 775-784, 2010.

16. Roderburg C, Urban GW, Bettermann K, et al: Micro-RNA profiling reveals a role for miR-29 in human and murine liver fibrosis. Hepatology 53: 209-218, 2011.
17. van Rooij E, Sutherland LB, Thatcher JE, et al: Dysregulation of microRNAs after myocardial infarction reveals a role of miR-29 in cardiac fibrosis. Proc Natl Acad Sci USA 105: 13027-13032, 2008.

18. Wang B, Komers R, Carew R, et al: Suppression of microRNA-29 expression by TGF- $\beta 1$ promotes collagen expression and renal fibrosis. J Am Soc Nephrol 23: 252-265, 2012.

19. Ye YM, Hu ZY, Lin Y, Zhang CF and Perez-Polo JR: Downregulation of microRNA-29 by antisense inhibitors and a PPAR- $\gamma$ agonist protects against myocardial ischaemia-reperfusion injury. Cardiovasc Res 87: 535-544, 2010.

20. Chang TC, Yu DN, Lee YS, et al: Widespread microRNA repression by Myc contributes to tumorigenesis. Nat Genet 40: 43-50, 2008.

21. Lagos-Quintana M, Rauhut R, Lendeckel W and Tuschl T: Identification of novel genes coding for small expressed RNAs. Science 294: 853-858, 2001.

22. Mourelatos Z, Dostie J, Paushkin S, et al: miRNPs: a novel class of ribonucleoproteins containing numerous microRNAs. Genes Dev 16: 720-728, 2002.

23. Landgraf $\mathrm{P}$, Rusu M, Sheridan R, et al: A mammalian microRNA expression atlas based on small RNA library sequencing. Cell 129: 1401-1414, 2007.

24. Yanaihara N, Caplen N, Bowman E, et al: Unique microRNA molecular profiles in lung cancer diagnosis and prognosis. Cancer Cell 9: 189-198, 2006.

25. Fabbri M, Garzon R, Cimmino A, et al: MicroRNA-29 family reverts aberrant methylation in lung cancer by targeting DNA methyltransferases 3A and 3B. Proc Natl Acad Sci USA 104: 15805-15810, 2007.

26. Plaisier CL, Pan M and Baliga NS: A miRNA-regulatory network explains how dysregulated miRNAs perturb oncogenic processes across diverse cancers. Genome Res 22: 2302-2314, 2012.

27. Cortez MA, Nicoloso MS, Shimizu M, et al: miR-29b and miR-125a regulate podoplanin and suppress invasion in glioblastoma. Genes Chromosomes Cancer 49: 981-990, 2010.

28. Xu H, Cheung IY, Guo HF and Cheung NKV: MicroRNA miR-29 modulates expression of immunoinhibitory molecule B7-H3: potential implications for immune based therapy of human solid tumors. Cancer Res 69: 6275-6281, 2009.

29. Namløs HM, Meza-Zepeda LA, Barøy T, et al: Modulation of the osteosarcoma expression phenotype by microRNAs. PLoS One 7: e48086, 2012.

30. Wang XH, Tang S, Le SY, et al: Aberrant expression of oncogenic and tumor-suppressive microRNAs in cervical cancer is required for cancer cell growth. PloS One 3: e2557, 2008.

31. Zhang W, Qian JX, Yi HL, et al: The microRNA-29 plays a central role in osteosarcoma pathogenesis and progression. Mol Biol 46: 622-627, 2012.

32. Ratert N, Meyer HA, Jung M, et al: Reference miRNAs for miRNAome analysis of urothelial carcinomas. PLoS One 7: e39309, 2012.

33. Wang G, Zhang $\mathrm{HH}, \mathrm{He} \mathrm{HD}$, et al: Up-regulation of microRNA in bladder tumor tissue is not common. Int Urol Nephrol 42: 95-102, 2010.

34. Ru P, Steele R, Newhall P, Phillips NJ, Toth K and Ray RB: miRNA-29b suppresses prostate cancer metastasis by regulating epithelial-mesenchymal transition signaling. Mol Cancer Ther 11: 1166-1173, 2012.

35. Resnick KE, Alder H, Hagan JP, Richardson DL, Croce CM and Cohn DE: The detection of differentially expressed microRNAs from the serum of ovarian cancer patients using a novel real-time PCR platform. Gynecol Oncol 112: 55-59, 2009.

36. Flavin R, Smyth P, Barrett C, et al: miR-29b expression is associated with disease-free survival in patients with ovarian serous carcinoma. Int J Gynecol Cancer 19: 641-647, 2009.

37. Hiroki E, Akahira J, Suzuki F, et al: Changes in microRNA expression levels correlate with clinicopathological features and prognoses in endometrial serous adenocarcinomas. Cancer Sci 101: 241-249, 2010.

38. Heinzelmann J, Henning B, Sanjmyatav J, et al: Specific miRNA signatures are associated with metastasis and poor prognosis in clear cell renal cell carcinoma. World J Urol 29: 367-373, 2011.

39. Garzon R, Garofalo M, Martelli MP, et al: Distinctive microRNA signature of acute myeloid leukemia bearing cytoplasmic mutated nucleophosmin. Proc Natl Acad Sci USA 105: 3945-3950, 2008.

40. Garzon R, Volinia S, Liu CG, et al: MicroRNA signatures associated with cytogenetics and prognosis in acute myeloid leukemia. Blood 111: 3183-3189, 2008. 
41. Calin GA, Ferracin M, Cimmino A, et al: A microRNA signature associated with prognosis and progression in chronic lymphocytic leukemia. N Engl J Med 353: 1793-1801, 2005.

42. Zhao JJ, Lin JH, Lwin T, et al: microRNA expression profile and identification of miR-29 as a prognostic marker and pathogenetic factor by targeting CDK6 in mantle cell lymphoma. Blood 115: 2630-2639, 2010.

43. Zanette DL, Rivadavia F, Molfetta GA, et al: miRNA expression profiles in chronic lymphocytic and acute lymphocytic leukemia. Braz J Med Biol Res 40: 1435-1440, 2007.

44. Sand M, Skrygan M, Sand D, et al: Expression of microRNAs in basal cell carcinoma. Br J Dermatol 167: 847-855, 2012.

45. Sengupta S, den Boon JA, Chen IH, et al: MicroRNA 29c is down-regulated in nasopharyngeal carcinomas, up-regulating mRNAs encoding extracellular matrix proteins. Proc Natl Acad Sci USA 105: 5874-5878, 2008.

46. Zeng X, Xiang JJ, Wu MH, et al: Circulating miR-17, miR-20a, miR-29c, and miR-223 combined as non-invasive biomarkers in nasopharyngeal carcinoma. PLoS One 7: e46367, 2012.

47. Wu Q, Wang C, Lu ZH, Guo L and Ge QY: Analysis of serum genome-wide microRNAs for breast cancer detection. Clin Chim Acta 413: 1058-1065, 2012.

48. Pass HI, Goparaju C, Ivanov S, et al: hsa-miR-29c* is linked to the prognosis of malignant pleural mesothelioma. Cancer Res 70: 1916-1924, 2010.

49. Fang C, Zhu DX, Dong HJ, et al: Serum microRNAs are promising novel biomarkers for diffuse large B cell lymphoma Ann Hematol 91: 553-559, 2012

50. Suzuki H, Saito Y and Hibi T: MicroRNAs in Gastric Cancer. Springer, New York, 2011.

51. Ding DP, Chen ZL, Zhao XH, et al: miR-29c induces cell cycle arrest in esophageal squamous cell carcinoma by modulating cyclin E expression. Carcinogenesis 32: 1025-1032, 2011.

52. Meng XZ, Zheng TS, Chen X, et al: microRNA expression alteration after arsenic trioxide treatment in HepG-2 cells. J Gastroenterol Hepatol 26: 186-193, 2011.

53. Xiong YJ, Fang JH, Yun JP, et al: Effects of microRNA-29 on apoptosis, tumorigenicity, and prognosis of hepatocellular carcinoma. Hepatology 51: 836-845, 2010.

54. Mott JL, Kobayashi S, Bronk SF and Gores GJ: mir-29 regulates Mcl-1 protein expression and apoptosis. Oncogene 26: 6133-6140, 2007.

55. Huang Z, Huang D, Ni S, Peng Z, Sheng W and Du X: Plasma microRNAs are promising novel biomarkers for early detection of colorectal cancer. Int J Cancer 127: 118-126, 2010.

56. Wang LG and Gu J: Serum microRNA-29a is a promising nove marker for early detection of colorectal liver metastasis. Cancer Epidemiol 36: E61-E67, 2012.

57. Sampath D, Liu CM, Vasan K, et al: Histone deacetylases mediate the silencing of miR-15a, miR-16, and miR-29b in chronic lymphocytic leukemia. Blood 119: 1162-1172, 2012.

58. Zhang XW, Zhao XH, Fiskus W, et al: Coordinated silencing of MYC-mediated miR-29 by HDAC3 and EZH2 as a therapeutic target of histone modification in aggressive B-cell lymphomas. Cancer Cell 22: 506-523, 2012

59. Mott JL, Kurita S, Cazanave SC, Bronk SF, Werneburg NW and Fernandez-Zapico ME: Transcriptional suppression of mir-29b-1/ mir-29a promoter by c-Myc, Hedgehog, and NF-kappaB. J Cell Biochem 110: 1155-1164, 2010.

60. Mishra A, Liu SJ, Sams GH, et al: Aberrant overexpression of IL-15 initiates large granular lymphocyte leukemia through chromosomal instability and DNA hypermethylation. Cancer Cell 22: 645-655, 2012

61. Wang H, Garzon R, Sun H, et al: NF-kappaB-YY1-miR-29 regulatory circuitry in skeletal myogenesis and rhabdomyosarcoma. Cancer Cell 14: 369-381, 2008.

62. Eyholzer M, Schmid S, Wilkens L, Mueller BU and Pabst T: The tumour-suppressive miR-29a/b1 cluster is regulated by CEBPA and blocked in human AML. Br J Cancer 103: 275-284, 2010.

63. Schmitt MJ, Philippidou D, Reinsbach SE, et al: Interferongamma-induced activation of signal transducer and activator of transcription 1 (STAT1) up-regulates the tumor suppressing microRNA-29 family in melanoma cells. Cell Commun Signal 10: 41,2012

64. Chou J, Lin JH, Brenot A, Kim JW, Provot S and Werb Z: GATA3 suppresses metastasis and modulates the tumour microenvironment by regulating microRNA-29b expression. Nat Cell Biol 15 201-213, 2013.

65. Melo SA and Kalluri R: miR-29b moulds the tumour microenvironment to repress metastasis. Nat Cell Biol 15: 139-140, 2013.
66. Muniyappa MK, Dowling P, Henry M, et al: MiRNA-29a regulates the expression of numerous proteins and reduces the invasiveness and proliferation of human carcinoma cell lines. Eur J Cancer 45: 3104-3118, 2009.

67. Garzon R, Liu SJ, Fabbri M, et al: MicroRNA-29b induces global DNA hypomethylation and tumor suppressor gene reexpression in acute myeloid leukemia by targeting directly DNMT3A and 3B and indirectly DNMT1. Blood 113: 6411-6418, 2009.

68. Li G, Zhao JF, Peng XJ, Liang J, Deng X and Chen YX: The mechanism involved in the loss of PTEN expression in NSCLC tumor cells. Biochem Biophys Res Commun 418: 547-552, 2012.

69. Wang XW, Zhao JF, Huang JH, Tang HH, Yu SY and Chen YX: The regulatory roles of miRNA and methylation on oncogene and tumor suppressor gene expression in pancreatic cancer cells. Biochem Biophys Res Commun 425: 51-57, 2012.

70. Bargaje R, Gupta S, Sarkeshik A, et al: Identification of novel targets for miR-29a using miRNA proteomics. PLoS One 7: e43243, 2012

71. Park SY, Lee JH, Ha M, Nam JW and Kim VN: miR-29 miRNAs activate p53 by targeting p85a and CDC42. Nat Struct Mol Biol 16: 23-29, 2009.

72. Garzon R, Heaphy CEA, Havelange V, et al: MicroRNA 29b functions in acute myeloid leukemia. Blood 114: 5331-5341, 2009.

73. Pekarsky Y, Santanam U, Cimmino A, et al: Tcl1 expression in chronic lymphocytic leukemia is regulated by miR-29 and miR-181. Cancer Res 66: 11590-11593, 2006.

74. Rothschild SI, Tschan MP, Federzoni EA, et al: MicroRNA-29b is involved in the Src-ID1 signaling pathway and is dysregulated in human lung adenocarcinoma. Oncogene 31: 4221-4232, 2012.

75. Wang C, Gao C, Zhuang JL, Ding C and Wang Y: A combined approach identifies three mRNAs that are down-regulated by microRNA-29b and promote invasion ability in the breast cancer cell line MCF-7. J Cancer Res Clin Oncol 138: 2127-2136, 2012

76. Cochrane DR, Jacobsen BM, Connaghan KD, Howe EN, Bain DL and Richer JK: Progestin regulated miRNAs that mediate progesterone receptor action in breast cancer. Mol Cell Endocrinol 355: 15-24, 2012

77. Lee TY, Ezelle HJ, Venkataraman T, Lapidus RG, Scheibner KA and Hassel BA: Regulation of human RNase-L by the miR-29 family reveals a novel oncogenic role in chronic myelogenous leukemia. J Interferon Cytokine Res 33: 34-42, 2013.

78. Gebeshuber CA,Zatloukal K and Martinez J: miR-29a suppresses tristetraprolin, which is a regulator of epithelial polarity and metastasis. EMBO Rep 10: 400-405, 2009.

79. Fang JH, Zhou HC, Zeng CX, et al: MicroRNA-29b suppresses tumor angiogenesis, invasion, and metastasis by regulating matrix metalloproteinase 2 expression. Hepatology 54: 1729-1740, 2011.

80. Price KJ, Tsykin A, Giles KM, et al: Matrigel basement membrane matrix influences expression of microRNAs in cancer cell lines. Biochem Biophys Res Commun 427: 343-348, 2012.

81. Wang F, Wang XS, Yang GH, et al: miR-29a and miR-142-3p downregulation and diagnostic implication in human acute myeloid leukemia. Mol Biol Rep 39: 2713-2722, 2012.

82. Li H, Solomon E, Muggy SD, Sun DQ and Zolkiewska A Metalloprotease-disintegrin ADAM12 expression is regulated by Notch signaling via microRNA-29. J Biol Chem 286: 21500-21510, 2011.

83. Zhi F, Zhou GX, Wang SN, et al: A microRNA expression signature predicts meningioma recurrence. Int J Cancer 132: 128-136, 2013

84. Weissmann-Brenner A, Kushnir M, Yanai GL, et al: Tumor microRNA-29a expression and the risk of recurrence in stage II colon cancer. Int J Oncol 40: 2097-2103, 2012.

85. Blum W, Garzon R, Klisovic RB, et al: Clinical response and miR-29b predictive significance in older AML patients treated with a 10-day schedule of decitabine. Proc Natl Acad Sci USA 107: 7473-7478, 2010

86. Blum W, Schwind S, Tarighat SS, et al: Clinical and pharmacodynamic activity of bortezomib and decitabine in acute myeloid leukemia. Blood 119: 6025-6031, 2012.

87. Visone R, Rassenti LZ, Veronese A, et al: Karyotype-specific microRNA signature in chronic lymphocytic leukemia. Blood 114: 3872-3879, 2009.

88. Zhu CL, Wang YG, Kuai WX, Sun XZ, Chen HP and Hong Z: Prognostic value of miR-29a expression in pediatric acute myeloid leukemia. Clin Biochem 46: 49-53, 2013. 